\title{
Improved Routing Protocol for Health Care Communications
}

\author{
Jayanthi K. Murthy ${ }^{1}$, V. Sambasiva Rao ${ }^{2}$ \\ ${ }^{1}$ Department of Electronics and Communication, B.M.S College of Engineering, Bengaluru, India \\ ${ }^{2}$ Department of Electronics and Communication, P.E.S Institute of Technology, Bengaluru, India \\ Email: jayanthimurthy2002@gmail.com,vsrao@pes.edu
}

Received February 23, 2013; revised March 29, 2013; accepted April 6, 2013

Copyright (c) 2013 Jayanthi K. Murthy, V. Sambasiva Rao. This is an open access article distributed under the Creative Commons Attribution License, which permits unrestricted use, distribution, and reproduction in any medium, provided the original work is properly cited.

\begin{abstract}
Wireless Body Area Network (WBAN) technology has significantly increased the potential of remote healthcare monitoring systems. The devices used for WBAN have limited energy resources. For most devices it is impossible to recharge or change the batteries. Since the data mostly consists of medical information, high reliability and low delay is required. The main objective of this simulation study is to evaluate the performance of routing protocol on static IEEE 802.15.4 to determine the most suitable routing protocol for Wireless Body Area Networks. Here, investigations on the various routing protocol suggest cluster topology and AODV as the probable candidate. About $16 \%$ improvement in the energy consumption was observed when modifications were made considering the energy and mobility, thus achieving high residual battery capacity and eliminating the need for recharging the batteries.
\end{abstract}

Keywords: Wireless Body Area Network; ZigBee Networks; Routing Protocol

\section{Introduction}

Advances in wireless communications, semiconductors and physiological sensing have given rise to miniature, lightweight, low power, intelligent monitoring devices. These devices can be integrated into a Wireless Body Area Network, a new enabling technology for monitoring health. The aging population in many developed countries and the rising costs of health care have triggered the development of novel technology-driven enhancements to current health care practices. Thus, the ubiquitous healthcare system focuses on early detection and prevention of chronic diseases, and provides an inexpensive and smart way to manage and care for patients suffering from age-related chronic diseases, such as heart disease which require continuous, long-term monitoring rather than sporadic assessments. Presently, continuous health monitoring system are wearable and easy to use consisting of tiny wireless sensors, strategically placed on the human body, creating a WBAN that monitors vital parameters and provide real-time feedback to the user and medical personnel. When integrated into a telemedicine system, they can even alert medical personnel about life-threatening changes. In addition, the wearable systems can be used for health monitoring of patients in am- bulatory settings [1]. A Wireless Body Area Network consists of small, intelligent devices attached on or implanted in the body which are capable of sampling, processing, and communicating one or more vital signs (heart rate, blood pressure, ECG, EEG, oxygen saturation,) or environmental parameters (temperature, humidity, location, light) and establishing a wireless communication link. These sensors are placed strategically on the human body as tiny patches or hidden in users' clothes allowing ubiquitous health monitoring for extended periods of time. These devices provide continuous health monitoring and real-time feedback to the user or medical personnel. The electronic medical records of registered users are maintained by the medical server which provides various services to the users and medical personnel. The server is responsible for authenticating users, accepting health monitoring session uploads, formatting and insertion of data into corresponding medical records, analysing the data patterns and recognizing serious health anomalies to help contact emergency services, or forward new instructions to the users. The physician can access the data from his/her office via the Internet, examine the reports to ensure the patient is within expected health metrics, ensure that the patient is responding to a given 
treatment or that a patient has been performing the prescribed exercises. The server agent is programmed to inspect the uploaded data and create an alert in the case of an emergency medical situation.

The sensor nodes used to monitor the vital statistics of the patient operate on batteries. The transmitted data mostly consists of medical information which has to be communicated efficiently to the medical server. Thus providing long battery life is the most critical parameter to be considered during design. The routing protocols play a significant role in transmitting the information. The routing protocols proposed for the Mobile Adhoc networks will not suit Body Area Networks as energy efficient communication is major concern here. WBANs are similar to MANETS in the sense that they have mobile nodes which need to reorganize themselves. But they differ in the number of nodes (usually only 10 - 15 nodes are present in WBANs) and the mobility speed. Thus routing protocols similar to the ones used in MANETs can be used. Two routing schemes are available in ZigBee networks, namely mesh routing and tree routing. The mesh routing scheme is similar to the Ad hoc On Demand Vector (AODV) routing algorithm, while the tree routing scheme resembles the cluster tree routing algorithm. The performance of the above mentioned algorithm has been extensively studied but previous evaluation studies are mostly IEEE 802.11 centric which consider all participating nodes to be capable of routing. Here, investigations are done on the various routing protocol to determine the most suitable routing protocol Wireless Body Area Networks.

\section{Overview of Zigbee}

ZigBee standard which is based on the IEEE 802.15.4 LR-WPAN standard has been proposed to interconnect simple, low rate, and battery powered wireless devices $[2,3]$. Numerous applications such as home-appliance networks, home healthcare, medical monitoring, military, consumer electronics, and environmental benefit from the deployment of ZigBee networks. The ZigBee specification establishes the framework for the Network and Application Layers based on the PHY and MAC layers [4] specified by IEEE 802.15.4 WPAN standard [6]. The PHY layer defines a total of 27 channels: 16 channels at a maximum rate of $250 \mathrm{kbps}$ in the ISM $2.4-2.4835$ $\mathrm{GHz}$ band, 10 channels at $40 \mathrm{kbps}$ in the ISM $902-928$ $\mathrm{MHz}$ band, and one channel at $20 \mathrm{kbps}$ in the 868.0 868.6 MHz band. At the MAC layer beaconless and beaconed modes access the radio channel using Carrier Sense Multiple Access with Collision Avoidance (CS MA/CA) or the optional slotted CSMA/CA mechanism. Two device types are specified within the IEEE 802.15.4 framework: full function device (FFD) that serves as net- work coordinators or network routers, and reduced function device (RFD) can only associate and communicate with FFDs in a ZigBee network. A FFD maintains routing tables, participate in route discovery and repair, maintains beaconing framework, and handle node joins. It also has the capability of communicating with any other devices within its transmission range. An RFD simply maintains the minimum amount of knowledge to stay on the network, and it does not participate in routing. RFDs can only associate and communicate with FFDs. FFDs and RFDs can be interconnected to form star or peer-to-peer networks. An effective routing scheme in a ZigBee network is important because it is the key to achieve efficiency in resource such as bandwidth and energy. Routing is not exactly similar to that in MANETs. In ZigBee Networks, FFD can serve as network coordinators or network routers while RFD can only associate and communicate with FFDs making them different from traditional MANET routing algorithms.

\section{Simulation Environment}

The main goal of this simulation is to analyze the performance of ZigBee using static IEEE 802.15.4 star topology for different existing routing protocols that can be used for health monitoring applications. Simulations are performed using QUALNET Version 5.0, software that provides scalable simulations of wireless networks. In the simulation model, one PAN Coordinator is deployed in an area of $5 \mathrm{~m} \times 5 \mathrm{~m}$. PAN is static main powered device placed at the centre of the simulation area. The transmission range of devices is one hop away from PAN Coordinator in star topology. In our simulation model, function to acknowledge the receipt of packets is disabled thus reducing the overhead. Only the uplink traffic i.e. devices to PAN Coordinator are considered in the simulations which suits WBAN application where a large number of devices communicates to a single sink for data delivery and processing. The simulation parameters are listed in Table 1. The Scenario for the WBAN shown in Figure 1 consists of one PAN coordinator and other 32 nodes sending packets to it.

The topology in the scenario contains four clusters each representing a single person with the nodes placed in specific locations and each cluster having a single identical cluster head. These cluster heads forward the data to the PAN coordinator. The number of nodes can be increased for critically ill patients.

Here, we consider the following five metric to determine the suitability of the protocols AODV [6], DYMO [7], ZRP [8] and OLSR [9] for health care applications.

Packet Delivery Ratio (PDR): is the rate of successfully delivering the data packets to the sink. It is denoted as $\mathrm{PRD}=(\mathrm{D} / \mathrm{S})^{*} 100$, Where $\mathrm{D}$ is the number of packets 
Table 1. Simulation parameters.

\begin{tabular}{ll}
\hline Parameters & Values \\
\hline No of Nodes & 33 \\
Terrain Area & $5 \mathrm{~m} \times 5 \mathrm{~m}$ \\
Path Loss Model & Two Ray Model \\
PHY and MAC Model & IEEE 802.15 .4 \\
Simulation Time & 60 mins \\
TX-Power & 0 dBM \\
Energy Model & MICAZ Mote \\
Battery Model & Simple Linear \\
Packet size & 50 bytes \\
Mobility & 0 to 5 mph \\
\hline
\end{tabular}

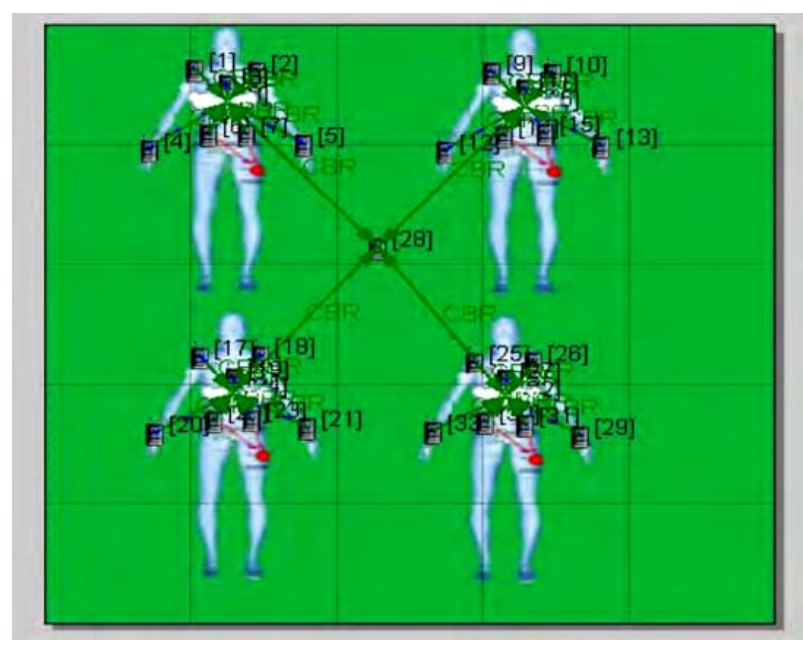

Figure 1. Scenario for WBAN.

received by the destination and $\mathrm{S}$ the number of packets sent by the source node.

Throughput: is the number of bits passed through a network in one second. It measures how fast data can pass through an entity (such as a point or a network). The throughput of a node is measured by counting the total number of data packets successfully received at the node and computing the number of bits received, which is finally divided by the total simulation runtime.

Throughput of a Node = $($ Total Data Bits Received $) /$ (Simulation Runtime).

The throughput of the network is defined as the average of the throughput of all nodes involved in data transmission.

Network Throughput $=($ Total throughput of nodes involved in data transmission)/(Number of nodes).

Energy Consumed: Energy is consumed in the active state when the nodes either transmit or receive and in the idle mode. Here the total energy consumed is the sum of transmitted and received energy.

Jitter: Jitter refers to a variation in packet delay, resulting in different packet inter-arrival times or out-ofsequence packets or both. It is often known as a measure of the variability over time of the packet latency across a network. A network with constant latency has no jitter. Packet jitter is expressed as an average of the deviation from the network mean latency.

Average End to End Delay: indicates the length of time taken for a packet to travel from the CBR (Constant Bit Rate) source to the destination. The average end-toend delay of a packet depends on delay at each hop comprising of queuing, channel access and transmission delays and route discovery latency.

Packet Delay $=($ Receive time at destination $)-($ Trans mit time at source)

Average Delay $=($ Sum of all packet delays $) /($ Total number of packets received)

\section{Energy Aware AODV Protocol (EAAODV)}

From the analysis of various routing protocols and based on the results of the performance of the protocols it was determined that AODV protocol performed the best. AODV protocol with cluster topology is well suited for Body Area Network application. Hence AODV is modified into an Energy aware routing protocol termed as EAAODV protocol. This routing protocol is an on-demand routing protocol which builds routes only on demand by flooding Route Request packets (RREQ) if a sender wishes to send data to a destination with no known route. Modification are made to the existing AODV protocol where in the each node maintains a routing table with the entries of its next hop neighbour, sequence number and hop count. The hop count is used to determine the distance to the destination and the sequence number measures the freshness of a route. The number of control overheads is reduced using efficient flooding technique. The selection of the neighbouring node for forwarding the route request is done based on mobility and the remaining energy. So if the node has mobility greater than a threshold, which is $5 \mathrm{mph}$, it is not selected as a neighbour. During route discovery from the source to the destination the energy values along the route are accumulated in the RREQ packets. At the destination or intermediate node (which has a fresh enough route to the destination) these values are copied into the Route Reply packet (RREP) which is transmitted back to the source. The source considers the maximum remaining energy capacity route and minimum mobility route every time it performs route discovery. This action will make the AODV routing protocol choose an alternative node or change the whole route to the destination node. 
Repeating this process can distribute the routing role among most of the nodes in the network, which is the focus of the proposed technique.

\section{Algorithm}

Step 1: Any wireless sensor node that wants to send a message must first look if a path exists from source to destination in the routing table.

If (route available): forward the message to next node Else message added to queue and source sends RREQ packet to neighbor to start the discovery process.

Let $\mathrm{E}=$ Energy and $\mathrm{M}=$ Mobility

Step 2: Learn the $\mathrm{E}$ and the $\mathrm{M}$ of the neighboring nodes and compare with that of the current node.

Step 3: Set a value of $E_{t h}$ and threshold mobility $M_{t h}$ depending on the application.

Step 4: If $E<E_{\text {th }}$ and $M>M_{\text {th, }}$ turn off the node and remove it from routing table

If $\mathrm{E}<\mathrm{E}_{\mathrm{th}}$ and $\mathrm{M}<\mathrm{M}_{\mathrm{th}}$, turn off the node for certain duration of time.

If $\mathrm{E}>\mathrm{E}_{\mathrm{th}}$ and $\mathrm{M}>\mathrm{M}_{\mathrm{th}}$, turn off the next hop for some time.

If $\mathrm{E}>\mathrm{E}_{\mathrm{th}}$ and $\mathrm{M}<\mathrm{M}_{\mathrm{th}}$, select the node for routing. Copy $\mathrm{E}$ and $\mathrm{M}$ into message.

Step 5: If destination node, calculate minimum mobility, maximum energy path. Write into RREP packet.

\section{Results and Discussion}

The performance comparison of AODV, DYMO, ZRP, OLSR protocol is presented in this section. Simulation experiments are carried out using QUALNET. Figure 2 shows the packet delivery ratio (PDR) of AODV, DYMO, ZRP, OLSR with and without mobility.

The average end to end delay which is a very important parameter to be considered in WBANs as critical data is transmitted to the medical server. Figure 3 shows that even when there is mobility, AODV protocol provides the best results followed by DYMO. Figure 4 demonstrates that mobility has a lot of effect in routing protocols operating in IEEE 802.15.4.Again AODV proves to be the one which provides minimum jitter an important parameter in QoS.

The energy consumed illustrates that throughput IERP is the highest both with and without mobility followed by ZRP minimum in AODV followed by OLSR as shown in Figures 5 and 6.

Tables 2 and $\mathbf{3}$ give the comparison of the various

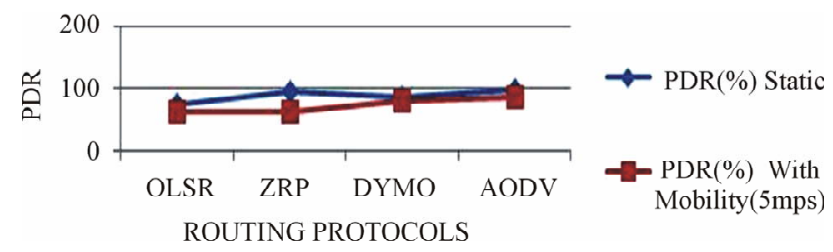

Figure 2. Comparison of \% PDR.

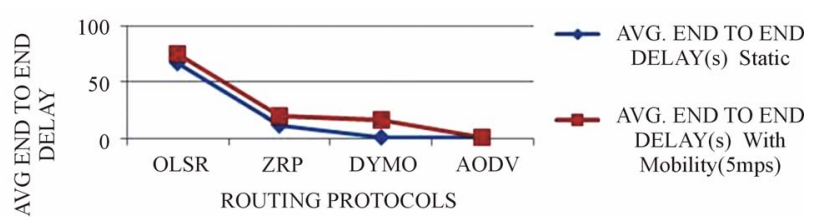

Figure 3. Comparison of average end to end delay.

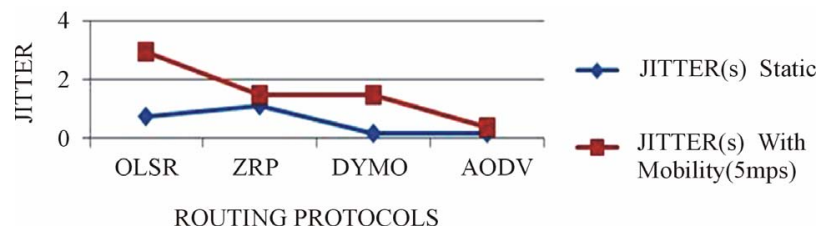

Figure 4. Comparison of jitter.

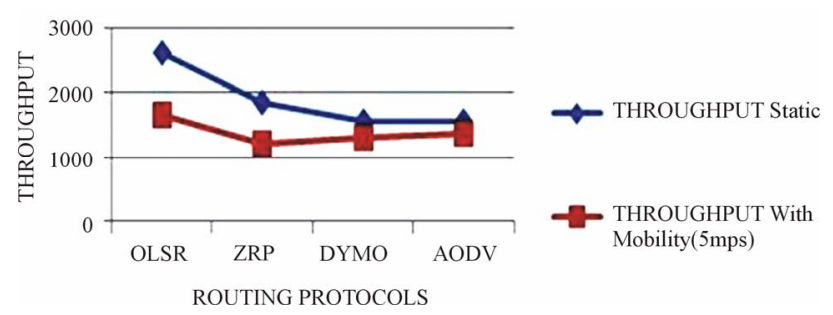

Figure 5. Comparison of throughput.

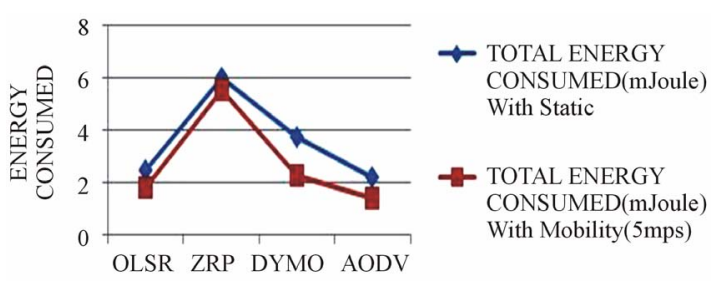

Figure 6. Energy consumed by various protocols.

protocols without and with mobility and based on the reading obtained we can say that AODV is the best protocol for nodes operating on ZigBee standard IEEE 802.15.4 when they are mobile.

Investigations on the different wireless mobile routing protocols like ZRP, AODV DYMO and OLSR have been made using CBR application in ZigBee network having static IEEE 802.15.4 star topology. Quality of service metrics (average end-to-end delay, throughput, jitter, and data packet delivery ratio) and energy consumption are used to compare to routing protocols. The findings suggest that AODV produces highest throughput with minimum jitter and delay with and without mobility. Therefore this routing protocol is the most suitable one for Wireless Body Area Networks. The main challenges in designing any efficient routing protocol is to address network partitioning with postural mobility, high propagation loss across the human body, low transmission power of the sensors, and low reliability of end-to-end path from source to sink. The parameters affecting network partitioning with postural mobility and residual 
Table 2. Comparison of parameters without mobility.

\begin{tabular}{cccccc}
\hline $\begin{array}{c}\text { routing } \\
\text { protocol }\end{array}$ & $\begin{array}{c}\text { through } \\
\text { put }\end{array}$ & delay & jitter & PDR & $\begin{array}{c}\text { energy } \\
\text { consumed }\end{array}$ \\
\hline OLSR & 2620 & 66.48 & 0.73 & 74.9 & 2.45 \\
ZRP & 1845 & 12.05 & 1.139 & 94.4 & 5.976 \\
DYMO & 1561 & 0.89 & 0.154 & 85 & 3.76 \\
AODV & 1583 & 0.96 & 0.17 & 97.5 & 2.24 \\
\hline
\end{tabular}

Table 3. Comparison of parameters with mobility.

\begin{tabular}{cccccc}
\hline $\begin{array}{c}\text { Routing } \\
\text { protocol }\end{array}$ & $\begin{array}{c}\text { through } \\
\text { put }\end{array}$ & delay & jitter & PDR & $\begin{array}{c}\text { energy } \\
\text { consumed }\end{array}$ \\
\hline OLSR & 1655 & 74.89 & 1.961 & 63.08 & 1.791 \\
ZRP & 1221 & 19.95 & 1.478 & 62.41 & 5.57 \\
DYMO & 1301 & 16.72 & 1.478 & 80.13 & 2.29 \\
AODV & 1390 & 1.47 & 0.3671 & 85.45 & 1.378 \\
\hline
\end{tabular}

energy are considered in the EAAODV protocol. Comparisons have been made with respect to packet delivery ratio, throughput and energy consumption.

Figure 7 shows that the Packet delivery ratio decreases for the heavy traffic due to collision of the packet leading to packet drop. When performance of the EAAODV and AODV are considered, both the results follow the same pattern for various traffic load but the packet delivery ratio of EAAODV is 12.73 percent higher than that of packet delivery ratio of AODV.

The throughput increases exponentially as the traffic load increases as shown in Figure 8. Compared to EAAODV, AODV has a throughput reduced by about 13.65 percent. Figure 9 shows the comparison of energy consumption. The energy consumption is more for light traffic and less for heavy traffic. Energy consumed decreases exponentially as the traffic load decreases. Only 89 percentage of the energy needed for AODV is sufficient for EAAODV in the transmit mode and only 87 percentage of the energy is needed to operate in receive mode. On the whole the energy consumed decreases to about 11.56 percent.

The total number of nodes was changed from 33 to see the variation of energy consumed when the number of

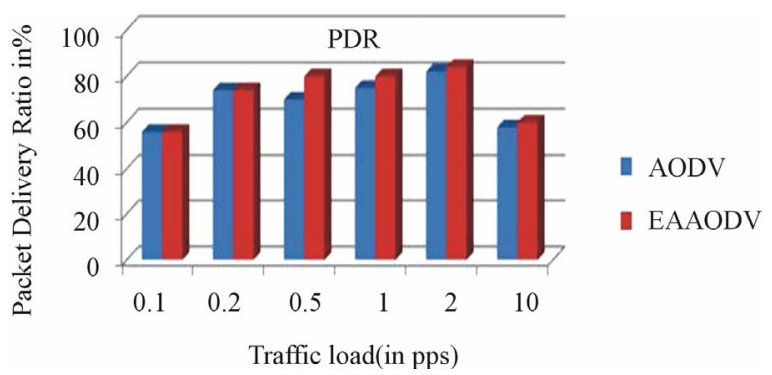

Figure 7. Comparison of packet delivery ratios. nodes was varied. Figure 10 shows that in comparison with AODV, EAAODV performs better with the total energy consumption being lower. This ensures that the proposed routing protocol provide better performance even when the network scales in size.

\section{Conclusion}

Investigations on wireless mobile routing protocols like ZRP, AODV, DYMO and OLSR using CBR application in ZigBee network having static IEEE 802.15.4 star topology have been made. Quality of service metrics (average end-to-end delay, throughput, jitter, and data packet delivery ratio) and energy consumption are used to compare to routing protocols. The findings suggest that AODV produces highest throughput with minimum jitter and delay with and without mobility. Therefore this routing protocol is the most suitable one for Wireless Body Area Networks. Thus, considering mobility and remaining energy as the criteria for selecting the nodes to

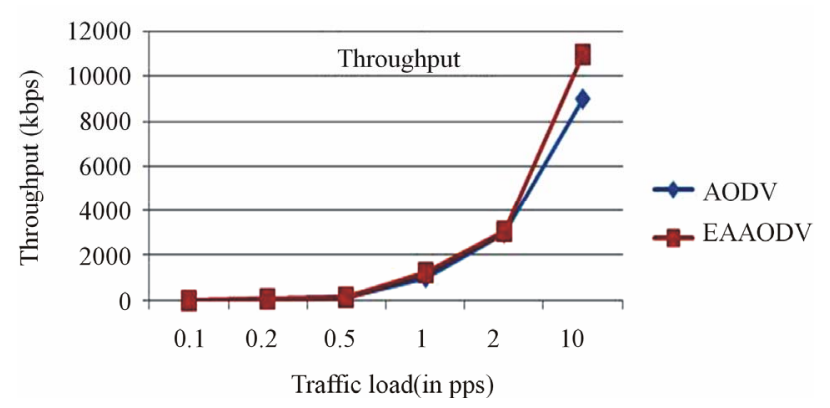

Figure 8. Comparison of throughput.

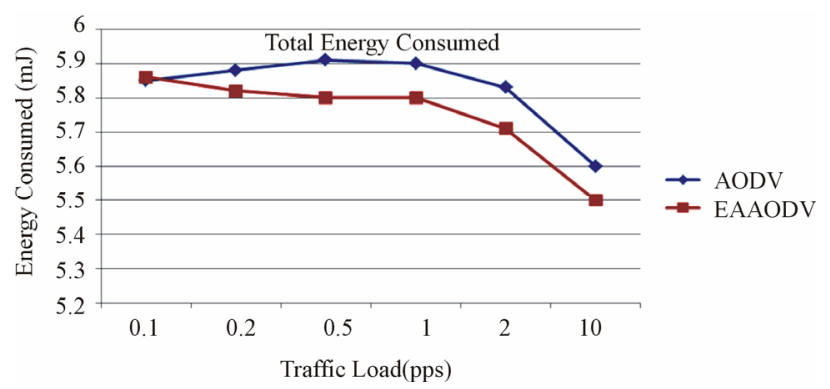

Figure 9. Comparison of total energy consumed.

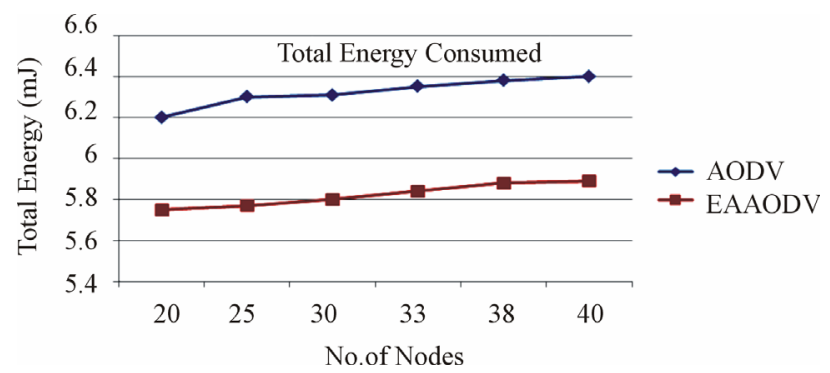

Figure 10. Comparison of total energy consumed with variation in number of nodes. 
forward the route request avoids unnecessary flooding. Thus the lifetime of the new protocol is better than the original AODV as the energy consumed is lesser.

\section{REFERENCES}

[1] S. Ullah, H. Higgins, B. Braem, B. Latre, C. Blondia, I. Moerman, S. Saleem, Z. Rahman and K. S. Kwak, "A Comprehensive Survey of Wireless Body Area Networks on PHY, MAC, and Network Layers Solutions,” Journal of Medical System, Vol. 36, No. 3, 2012, pp. 1065-1094.

[2] A. Bhatia and P. Kaushik, "A Cluster Based Minimum Battery Cost AODV Routing Using Multipath Route for Zigbee,” ICON 2008 16th IEEE International Conference on Networks, New Delhi, 12-14 December 2008, pp. 1-7.

[3] N. Golmie, D. Cypher and O. Rebala, "Performance Analysis of Low Rate Wireless Technologies for Medical Applications," Computer Communications, Vol. 28, No. 10, 2005, pp. 1266-1275.

[4] S. Mohanty, "Energy Efficient Routing Algorithms for Wireless Sensor Networks and Performance Evaluation of

\section{Abbreviations Used}

Wireless Body Area Network (WBAN),

Ad hoc On Demand Vector (AODV),

Electrocardiogram (ECG),

Electroencephalogram (EEG),

Mobile Ad hoc networks (MANETS), (LR-WPAN),

Physical (PHY), Medium Access Layer (MAC),

Carrier Sense Multiple Access with Collision Avoidance

(CSMA/CA),
Quality of Service for IEEE 802.15.4 Networks,” Master's thesis, National Institute of Technology, Rourkela, Orissa, 2010.

[5] IEEE 802.15.4: Mac and Physical Specifications for lRPANS, 2003. http://www.ieee802.org/15/pub/TG4

[6] C. Perkins, E. Royer and S. Das, "Ad hoc On Demand Distance Vector (AODV) Routing,” The Zone Routing Protocol, Internet Draft,1999.

[7] A. S. Raghuvanshi and S. Tiwari, "DYMO as Routing Protocol for IEEE-802.15.4 Enabled Wireless Sensor Networks," IEEE 2010 6th International Conference on Wireless Communication and Sensor Networks (WCSN), Allahabad, 15-19 December 2010, pp. 1-6.

[8] R. M. Pearlman and J. Z. Haas, "Determining the Optimal Configuration for the Zone Routing Protocol," IEEE Journal on Selected Areas in Communications, Vol. 17, No. 8, 1999, pp. 1395-1414.

[9] T. Clausen and P. Jacquet, “Optimized Link State Routing Protocol (OLSR),” RFC 3626, IETF, Network Working Group, 2003. full function device (FFD),

reduced function device (RFD),

Personal Access Network ( PAN),

Zone Routing Protocol (ZRP),

Optimized Link State Routing Protocol (OLSR),

Dynamic MANET On-demand (DYMO),

Packet Delivery Ratio (PDR). 